08

\title{
Оптимизация состава, синтез и изучение широкополосных многослойных зеркал для ЭУФ диапазона
}

\author{
( М.М. Барышева, ${ }_{1}$ С.А. Гарахин, ${ }^{1}$ C.Ю. Зуев, ${ }^{1}$ В.Н. Полковников, ${ }^{1}$ Н.Н. Салащенко, ${ }^{1}$ М.В. Свечников, ${ }^{1}$ \\ P.М. Смертин, ${ }^{1}$ Н.И. Чхало, ${ }^{1}$ E. Meltchakov ${ }^{2}$ \\ ${ }^{1}$ Институт фризики микроструктур РАН, \\ 607680 Нижний Новгород, Россия \\ ${ }^{2}$ Laboratoire Charles Fabry, Institut d'Optique Graduate School, \\ 91127 Palaiseau, France \\ e-mail: mmbarysheva@ipmras.ru
}

Поступило в Редакцию 28 марта 2019 г.

В окончательной редакции 28 марта 2019 г.

Принято к публикации 15 апреля 2019 г.

Разработаны и изготовлены широкополосные $\mathrm{Mo} / \mathrm{Si}$ и $\mathrm{Mo} / \mathrm{Be}$ многослойные зеркала стекового типа для диапазонов длин волн $11.1-13.8,17-21$ и $28-33 \mathrm{~nm}$. Показано, что для таких структур равномерное отражение может быть достигнуто за небольшое число коррекций технологического процесса.

Ключевые слова: ЭУФ, широкополосные зеркала, апериодические зеркала, стековые структуры, рефлектометр с лазерно-плазменным источником.

DOI: 10.21883/JTF.2019.11.48341.116-19

\section{Введение}

Многослойные рентгеновские зеркала с изменяющимся по глубине периодом и уширенной спектральной или угловой кривой отражения широко применяются в рентгенооптических исследованиях [1]. В солнечной астрономии они являются рабочим элементом спектрогелиографов [2], в спектроскопии лабораторной плазмы [3] позволяют собрать на порядок больше информации от исследуемого объекта благодаря высокому интегральному коэффициенту отражения; актуальны как элементы для транспортировки, коллимации и фокусировки аттосекундных импульсов электромагнитного излучения [4].

В настоящий момент разработано два принципиально разных подхода к дизайну многослойных зеркал, обладающих максимальным равномерным отражением в заданном спектральном диапазоне. В обоих случаях в качестве отправной точки берется периодическое многослойное зеркало (ПМЗ), образованное тонкими чередующимися слоями материалов с разной диэлектрической проницаемостью, однако „период“ структуры изменяется по глубине. В случае апериодической структуры (АМЗ) все слои имеют индивидуальную толщину. Распределение толщин в жестком рентгеновском диапазоне подчиняется степенному закону [5-7], или хаотическому, рассчитываемому численно $[8,9]$ - в мягком рентгеновском и экстремальном ультрафиолетовом (ЭУФ) диапазонах. Второй подход, так называемый стековый [10] (от английского stack): многослойное зеркало представляет собой несколько напыленных одно на другое ПМЗ с разными характеристиками: $\left\{N_{i}, d_{i}, \gamma_{i}\right\}$ количество периодов, величина периода, доля сильнопо- глощающего вещества соответственно $(i-$ номер ПМЗ в составе стековой структуры). С увеличением числа ПМЗ и $N_{i}=1$ такая структура, очевидно, сводится к АМЗ. Отсюда же следует неизбежно меньшая гладкость кривой отражения оптимизированной стековой структуры и несколько меньшие коэффициенты отражения: при оптимизации состава АМЗ должен быть найден компромисс между гладкостью „полочки“ и ее высотой.

Плюсы стекового подхода определяются, прежде всего, технологией. Фактически, чтобы корректно напылить $N$ различных толщин, необходимо провести порядка $N$ калибровок, что в случае АМЗ с большим (несколько десятков) числом слоев оказывается чрезвычайно трудозатратным. Что касается решения обратной задачи, для классического АМЗ она практически нерешаема из-за большого числа параметров и неоднозначности полученного решения. В случае стекового многослойного зеркала (СМЗ) число восстанавливаемых параметров существенно меньше, обратная задача может быть достоверно решена, и проведена соответствующая коррекция технологического процесса. Приведенные соображения объясняют преимущество использования стекового дизайна при изготовлении широкополосных рентгеновских зеркал.

В ИФМ РАН имеется опыт изготовления апериодических широкополосных зеркал на основе пар материалов $\mathrm{Mo} / \mathrm{Si}$ и $\mathrm{Mo} / \mathrm{Be}$ для космической аппаратуры исследования солнца КОРТЕС $[9,11]$. Настоящая работа посвящена разработке и изготовлению стековых структур с равномерным отражением в диапазонах длин волн $11.1-13.8$, $17-21$ и $28-33 \mathrm{~nm}$. 


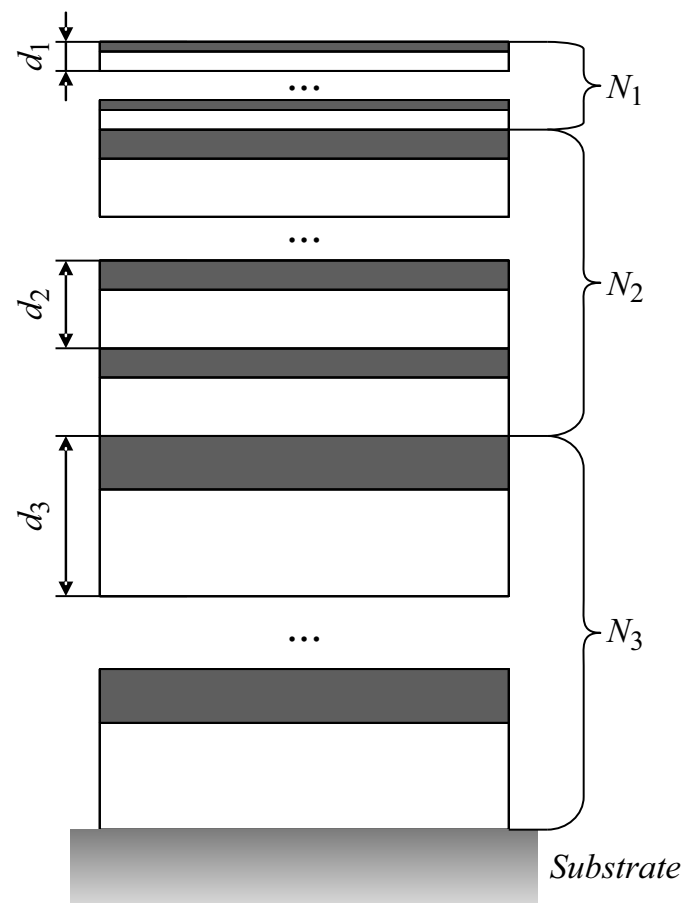

Рис. 1. Схема многослойного зеркала стекового типа.

\section{1. Оптимизация состава стековых структур}

Традиционной парой материалов, применяемой в диапазоне $12.5-35 \mathrm{~nm}$ является $\mathrm{Mo} / \mathrm{Si}$, однако в области $12.3-12.4 \mathrm{~nm}$ расположен край поглощения $\mathrm{Si}$ и в качестве „спейсера“ в этой области применяется Ве. Таким образом, в диапазонах 17-21 и 28-33 nm были рассчитаны и изготовлены Mo/Si стековые зеркала, в диапазоне $11.1-13.8 \mathrm{~nm}$ - Mo/Be, как обладающие наибольшим экспериментально полученным коэффициентом отражения в этой области [12-14].

Схематическое изображение стековой структуры, состоящей из трех МПЗ, приведено на рис. 1. Поиск параметров СМ3, обеспечивающих равномерное отражение в заданном диапазоне длин волн, представляет собой задачу многомерной оптимизации, сводящуюся к минимизации функционала

$$
F=\int\left[R(\lambda)-R^{\text {target }}\right]^{2 m} d \lambda
$$

где $R(\lambda)$ - зависимость коэффициента отражения от длины волны, $R^{\text {target }}$ — целевая функция для коэффициента отражения (определяет высоту „плато“), интеграл вычисляется в области определения $R^{\text {target }}$. Процедура содержит несколько итераций, вначале высота „Плато“ выбирается на уровне, соответствующем отражению ПМЗ, и постепенно понижается до достижения удовлетворительной гладкости.

В качестве параметров оптимизации рассматривается набор $\left\{N_{i}, d_{i}, \gamma_{i}\right\}$, в случае использования защитного покрытия или прослоек к варьируемым параметрам добавляются их толщины. Плотности материалов и характеристики интерфейсов считаются известными из экспериментов с периодическими зеркалами. Так, для пары Mo/Si ширины переходных областей, понимаемых в смысле среднеквадратичной шероховатости $\sigma$, составляют $\sigma=1.2 \mathrm{~nm}$ (Мо-на- $\mathrm{Si}$ ) и $\sigma=0.6 \mathrm{~nm}$ ( $\mathrm{Si}-$-на-Mo) [13], плотности пленок табличные. Случай $\mathrm{Mo} / \mathrm{Be}$ изучен хуже [14-16], при расчетах мы закладывали $\sigma=0.7 \mathrm{~nm}$ (Мо-на-Ве) и $\sigma=0.3 \mathrm{~nm}$ (Ве-на-Мо). Как показано в [9], в случае апериодических зеркал результат решения задачи оптимизации будет существенно разным для нулевых и ненулевых значений $\sigma$, поэтому важно изначально учитывать реалистичные параметры переходных областей. Анализ, однако, показывает, что незначительное (порядка $\AA$ ) отличие величин шероховатостей не должно приводить к драматическому искажению кривой отражения, т.е. строгое выполнение приведенных выше соотношений не необходимо.

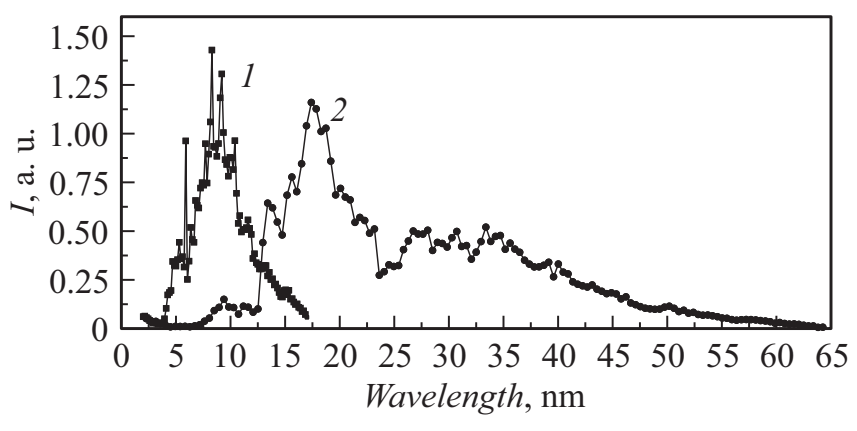

Рис. 2. Спектры источника излучения ЭУФ диапазона, промеренные дифракционными решетками 900 (1) и 300 (2) lines/mm.

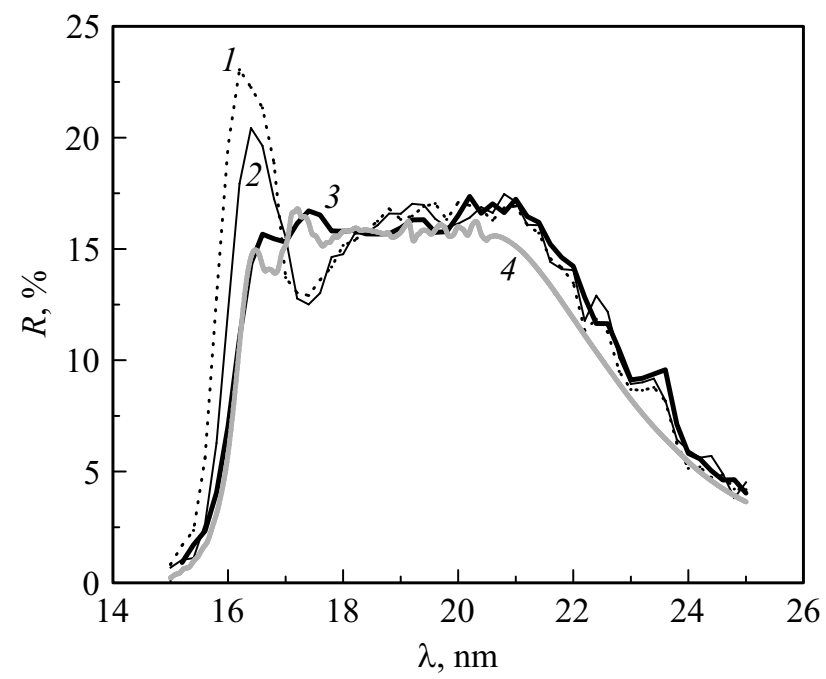

Рис. 3. Экспериментально полученные $(1-3-$ номера итераций) и расчетная (4) кривые отражения $\mathrm{Mo} / \mathrm{Si} \mathrm{CM} 3$, оптимизированных на равномерное отражение в диапазоне $17-21 \mathrm{~nm}$. 
Таблица 1. Расчетные и восстановленные толщины слоев Mo/Si CM3 для диапазона 17-21 nm

\begin{tabular}{|c|c|c|c|c|c|c|}
\hline \multirow{2}{*}{$\begin{array}{c}\text { Номер ПМЗ } \\
\text { от } \\
\text { поверхности }\end{array}$} & \multirow{2}{*}{$\begin{array}{c}\text { Число } \\
\text { периодов, } \\
N_{i}\end{array}$} & \multirow{2}{*}{ Материал } & \multicolumn{4}{|c|}{ Толщины слоев ПМЗ,nm } \\
\hline & & & Расчет & $\begin{array}{c}\text { Первичная } \\
\text { структура }\end{array}$ & $\begin{array}{c}\text { 1-я } \\
\text { коррекция }\end{array}$ & $\begin{array}{l}\text { Финальная } \\
\text { коррекция }\end{array}$ \\
\hline пленка $\mathrm{Si}$ & 1 & $\mathrm{Si}$ & 1.40 & 1.40 & 1.40 & 1.40 \\
\hline 1 & 3 & $\begin{array}{c}\mathrm{Mo} \\
\mathrm{Si}\end{array}$ & $\begin{array}{l}4.30 \\
6.72\end{array}$ & $\begin{array}{l}4.34 \\
6.66\end{array}$ & $\begin{array}{l}4.33 \\
6.66\end{array}$ & $\begin{array}{l}4.34 \\
6.72\end{array}$ \\
\hline 2 & 3 & $\begin{array}{c}\text { Mo } \\
\text { Si }\end{array}$ & $\begin{array}{l}3.43 \\
5.37\end{array}$ & $\begin{array}{l}3.35 \\
5.21\end{array}$ & $\begin{array}{l}3.35 \\
5.21\end{array}$ & $\begin{array}{l}3.44 \\
5.39\end{array}$ \\
\hline 3 & 32 & $\begin{array}{c}\mathrm{Mo} \\
\mathrm{Si}\end{array}$ & $\begin{array}{l}4.50 \\
4.50\end{array}$ & $\begin{array}{l}4.30 \\
4.30\end{array}$ & $\begin{array}{l}4.40 \\
4.40\end{array}$ & $\begin{array}{l}4.52 \\
4.52\end{array}$ \\
\hline
\end{tabular}

Таблица 2. Расчетные и восстановленные параметры Mo/Si CM3 для диапазона 28-33 nm

\begin{tabular}{|c|c|c|c|c|}
\hline \multirow{2}{*}{$\begin{array}{l}\text { Номер ПМЗ от } \\
\text { поверхности }\end{array}$} & \multirow{2}{*}{$\begin{array}{c}\text { Число } \\
\text { периодов, } N\end{array}$} & \multirow{2}{*}{ Материал } & \multicolumn{2}{|c|}{ Толщины слоев ПМЗ,nm } \\
\hline & & & Расчет & Эксперимент \\
\hline пленка Si & 1 & $\mathrm{Si}$ & 5.3 & 7.6 \\
\hline 1 & 2 & $\begin{array}{c}\mathrm{Mo} \\
\mathrm{Si}\end{array}$ & $\begin{array}{r}5.58 \\
10.36\end{array}$ & $\begin{array}{r}4.81 \\
10.99\end{array}$ \\
\hline 2 & 1 & $\begin{array}{c}\mathrm{Mo} \\
\mathrm{Si}\end{array}$ & $\begin{array}{l}10.57 \\
12.5\end{array}$ & $\begin{array}{l}11.12 \\
13.09\end{array}$ \\
\hline 2 & 5 & $\begin{array}{c}\mathrm{Mo} \\
\mathrm{Si}\end{array}$ & $\begin{array}{l}5.62 \\
12.5\end{array}$ & $\begin{array}{r}5.45 \\
12.49\end{array}$ \\
\hline 2 & 1 & $\begin{array}{c}\mathrm{Mo} \\
\mathrm{Si}\end{array}$ & $\begin{array}{r}5.62 \\
15.21\end{array}$ & $\begin{array}{r}6.63 \\
16.49\end{array}$ \\
\hline 3 & 20 & $\begin{array}{c}\mathrm{Mo} \\
\mathrm{Si}\end{array}$ & $\begin{array}{r}4.95 \\
13.38\end{array}$ & $\begin{array}{r}4.68 \\
13.20\end{array}$ \\
\hline
\end{tabular}

Минимизация (1) осуществлялась при помощи алгоритма дифференциальной эволюции, реализованного в рамках программы Multifitting [17], в отличие от широко распространенной IMD [18] позволяющей рассматривать $N_{i}$ в качестве параметра оптимизации. Мы не накладывали априорных ограничений на характер изменения $d_{i}$ вглубь структуры, устанавливая на первом шаге достаточно широкий (избыточный с точки зрения условия Брэгга) и одинаковый для всех $d_{i}$ диапазон изменения (например, 5-100 nm при диапазоне $28-33 \mathrm{~nm}$ ). Из всех решений, соответствующих минимуму (1), оптимальными считались параметры, обеспечивающие максимальную гладкость кривой отражения в заданном спектральном интервале при максимально доступной высоте „Полочки“.

Параметры рассчитанных СМЗ и соответствующие им кривые отражения можно видеть в таблицах 1-3 и на рисунках 3-7. Во всех приведенных случаях рассчитанные стековые структуры демонстрируют рост $d_{i}$ к подложке аналогично $[10,19]$ (отметим, что в ,жестком“ диапазоне наблюдается другая картина [20]). При этом верхние слои играют роль фильтра, подавляющие межстековые отражения и ослабляющие осцилляции кривой отражения зеркала.

\section{2. Метод напыления}

Образцы изготавливались методом магнетронного распыления в атмосфере аргона при давлении $1 \cdot 10^{-3}$ Torr; в качестве подложек использованы кремниевые пластины для микроэлектронной промышленности с эффективной шероховатостью в диапазоне пространственных частот $0.024-65 \mu \mathrm{m}^{-1} \sigma=0.3 \mathrm{~nm}$ [21]. Подробное описание напылительной установки содержится в [22]. Толщина осажденной пленки материала определяется временем прохождения подложки над мишенью и величиной тока на магнетроне, поэтому процессу изготовления зеркала предшествует процедура калиб- 


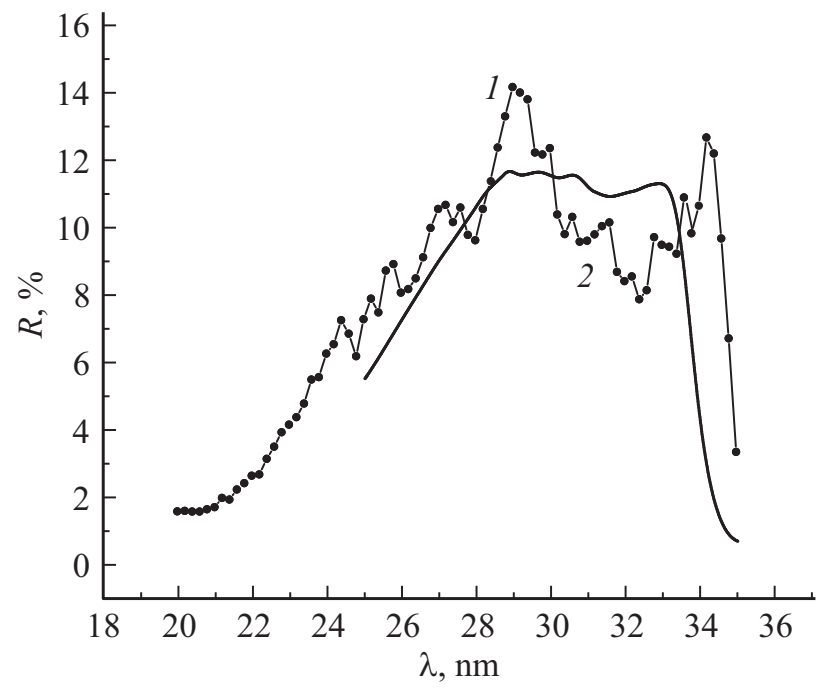

Рис. 4. Экспериментально полученная (1) и расчетная (2) кривые отражения Mo/Si CM3, оптимизированных на равномерное отражение в диапазоне $28-33 \mathrm{~nm}$ (первая итерация).

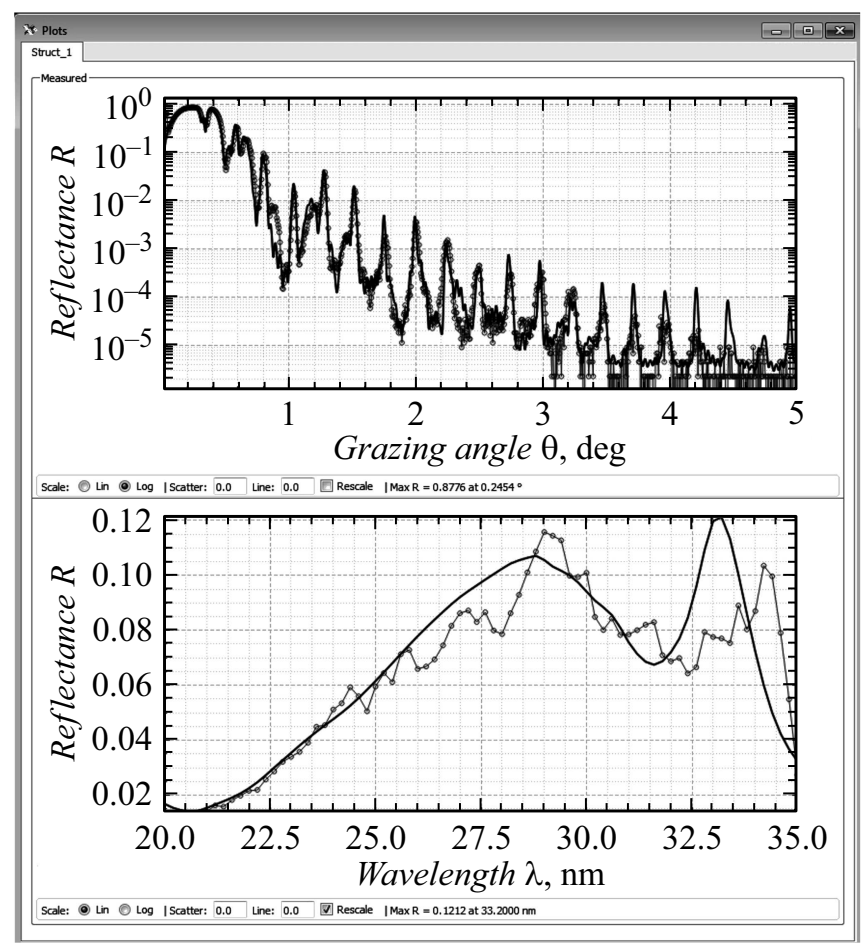

Рис. 5. Окно программы Multifitting: одновременная подгонка экспериментальных кривых отражения при $\lambda=0.154 \mathrm{~nm}$ и $20-35 \mathrm{~nm} \mathrm{Mo/Si} \mathrm{CM3} \mathrm{для} 28-33 \mathrm{~nm}$, теоретический расчет показан сплошной линией.

ровки. Однако неизбежный дрейф режима в установке, микропробои, незначительные изменения давления рабочего газа могут приводить к отклонению толщин пленок от заданных значений. Процедура изготовления многослойного покрытия, таким образом - итераци- онная, требующая определения параметров зеркала и соответствующей коррекции процесса напыления.

\section{3. Характеризация структур}

Для контроля за технологическим процессом с целью его коррекции проводилась характеризация образцов методом малоугловой дифракции рентгеновских

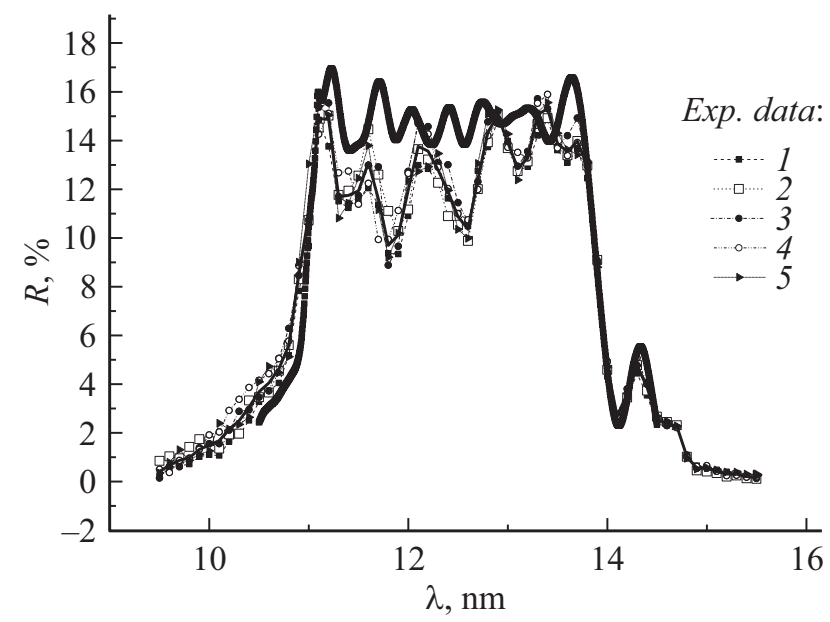

Рис. 6. Экспериментально полученные усреднением по пяти реализациям (1) и расчетная (2) кривые отражения Мо/Be СМ3, оптимизированных на равномерное отражение в диапазоне $11.1-13.8 \mathrm{~nm}$ (сплошные линии).

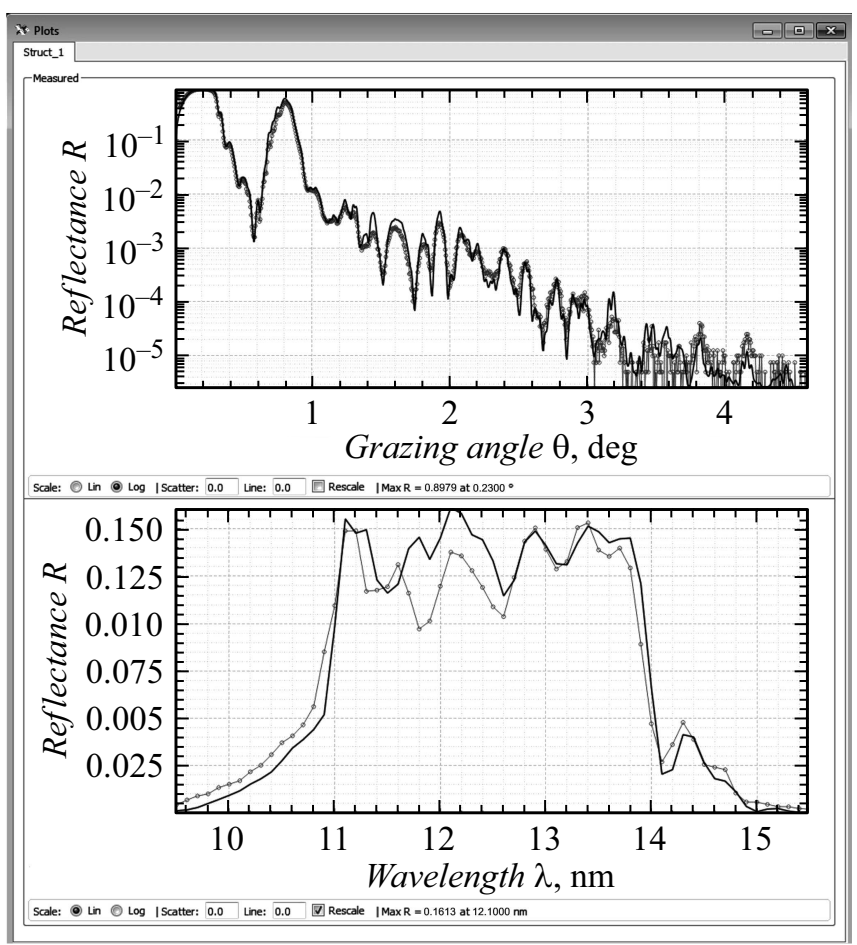

Pис. 7. Окно программы Multifitting: одновременная подгонка экспериментальных кривых отражения при $\lambda=0.154 \mathrm{~nm}$ и 9.6-15.2 nm Мo/Be СМЗ для $11.1-13.8 \mathrm{~nm}$, теоретический расчет показан сплошной линией. 
Таблица 3. Расчетные и восстановленные параметры Мо/Ве СМЗ для диапазона $11.1-13.8 \mathrm{~nm}$

\begin{tabular}{|c|c|c|c|c|}
\hline \multirow{2}{*}{$\begin{array}{c}\text { Номер ПМЗ от } \\
\text { поверхности }\end{array}$} & \multirow{2}{*}{$\begin{array}{c}\text { Число } \\
\text { периодов, } N\end{array}$} & \multicolumn{3}{|c|}{ Толщины слоев ПМЗ,nm } \\
\hline & & Материал & Расчет & Эксперимент \\
\hline 1 & 2 & $\begin{array}{l}\text { Be } \\
\text { Mo }\end{array}$ & $\begin{array}{l}2.72 \\
2.22\end{array}$ & $\begin{array}{c}\text { BeO: } 1.43 \\
\text { Be: } 2.93 \\
\text { Mo: } 2.22 \\
\text { Be: } 26.8 \\
\text { Mo: } 2.26\end{array}$ \\
\hline 2 & 4 & $\begin{array}{l}\mathrm{Be} \\
\mathrm{Mo}\end{array}$ & $\begin{array}{l}3.38 \\
2.25\end{array}$ & $\begin{array}{l}3.32 \\
2.31\end{array}$ \\
\hline 3 & 6 & $\begin{array}{l}\mathrm{Be} \\
\mathrm{Mo}\end{array}$ & $\begin{array}{l}2.95 \\
3.07\end{array}$ & $\begin{array}{l}2.87 \\
3.19\end{array}$ \\
\hline 4 & 8 & $\begin{array}{l}\text { Be } \\
\text { Mo }\end{array}$ & $\begin{array}{l}3.08 \\
3.33\end{array}$ & $\begin{array}{l}3.02 \\
3.41\end{array}$ \\
\hline 5 & 11 & $\begin{array}{l}\mathrm{Be} \\
\mathrm{Mo}\end{array}$ & $\begin{array}{l}3.20 \\
3.75\end{array}$ & $\begin{array}{l}3.18 \\
3.82\end{array}$ \\
\hline 3 & 6 & $\begin{array}{l}\mathrm{Be} \\
\mathrm{Mo}\end{array}$ & $\begin{array}{l}2.95 \\
3.07\end{array}$ & $\begin{array}{l}2.87 \\
3.19\end{array}$ \\
\hline 4 & 8 & $\begin{array}{l}\mathrm{Be} \\
\mathrm{Mo}\end{array}$ & 3.08 & 3.02 \\
\hline 6 & 10 & $\begin{array}{l}\mathrm{Be} \\
\mathrm{Mo}\end{array}$ & $\begin{array}{l}3.05 \\
4.38\end{array}$ & $\begin{array}{l}2.93 \\
4.54\end{array}$ \\
\hline
\end{tabular}

лучей на длине волны $\mathrm{Cu} K_{\alpha} 0.154 \mathrm{~nm}$ с использованием дифрактометра PANalitycal X'Pert Pro. Дифрактометр оснащен четырехкристальным ассиметричным монохроматором $\mathrm{Ge}(220)$; образец закрепляется на столе с 6-ю степенями свободы, механика прибора обеспечивает точность установки углов на уровне $0.001^{\circ}$. Измерения проводились в диапазоне углов скольжения излучения на образец $\theta=0-5^{\circ}$, доступный динамический диапазон по интенсивности рентгеновского пучка составляет $10^{6}$.

В рабочем ЭУФ диапазоне широкополосные зеркала изучаются на разработанном в ИФМ РАН рефлектометpe, в котором монохроматизация излучения осуществляется с помощью высокоразрешающего спектрометра Черни-Тюрнера с плоской дифракционной решеткой и двумя сферическими коллимирующими зеркалами и лазерно-плазменным источником излучения [23]. В отличие от традиционно применяемых спектрометровмонохроматоров роуландовского типа такая схема, обладая высоким спектральным разрешением и широким рабочим диапазоном, сохраняет входную и выходную щели неподвижными, практически полностью снимая ограничения на массогабаритные характеристики источника излучения. Рефлектометр оснащен пятиосным гониометром, позволяющим изучать многослойные зеркала, нанесенные на криволинейные подложки.

Источником рентгеновского излучения в описываемом рефлектометре является высокоионизованная плаз- ма, генерируемая при взаимодействии мощного лазерного пучка (плотность мощности $10^{11}-10^{12} \mathrm{~W} / \mathrm{cm}^{2}$ ) на твердотельную мишень. Для стабилизации эмиссионных характеристик необходимо избегать образования глубоких кратеров на мишени, что достигается ее постоянным смещением, поступательным линейным и вращательным. Сканирование по спектру осуществляется поворотом дифракционной решетки; калибровка спектра производится по краям поглощения свободновисящих фильтров $\mathrm{Be}, \mathrm{Al}, \mathrm{MoZrSi}_{2}, \mathrm{MoSi}_{2}$ и $\mathrm{ZrSi}$. Монохроматический зондовый пучок падает на тороидальное зеркало, формирующее на образце зондовый пучок размером $0.29 \times 0.16 \mathrm{~mm}$. Расходимость пучка по горизонтали (в плоскости сканирования) составила $0.008^{\circ}$, по вертикали $-0.07^{\circ}$.

Спектры источника с мишенью из нержавеющей стали измерены в диапазоне 4-60 nm (рис. 2) с использованием дифракционных решеток 900 и $300 \mathrm{lines} / \mathrm{mm}$ (спектральное разрешение 0.03 и $0.06 \mathrm{~nm}$ соответственно). Первая решетка позволяет эффективно работать в диапазоне 5-15 nm, вторая - 9-24 nm, а при дополнительном использовании Al-фильтра для отсечения второго порядка дифракции - вплоть до $34 \mathrm{~nm}$. Для оценки точности измерений рефлектометра в диапазоне длин волн $11-14 \mathrm{~nm}$ было проведено сравнение спектральных и угловых зависимостей коэффициентов отражения $\mathrm{Mo} / \mathrm{Si}$ и 
Мо/Ве зеркал с результатами, полученными на BESSY-2, которые показали совпадение в пределах $\pm 1.5 \%$.

На основе данных, полученных в жестком и мягком рентгеновских диапазонах, решается обратная задача, для чего применяется авторская программа „Multifitting“ [18], позволяющая одновременно производить подгонку нескольких кривых отражения, полученных в разных частотных диапазонах. В качестве параметров подгонки выступают характеристики стеков, составляющих широкополосное зеркало: толщины и плотности материалов, шероховатости/масштабы переходных областей. После восстановления параметров реальной структуры, отличных от теоретически рассчитанных, производится коррекция процесса напыления.

\section{4. Экспериментальные результаты и обсуждение}

В рамках настоящей работы были синтезированы и изучены образцы широкополосных Мо-содержащих CM3, изготовленных по описанной выше схеме и оптимизированных на равномерное отражение в диапазонах длин волн $17-21,28-33,11.1-13.8 \mathrm{~nm}$.

$\mathrm{Mo} / \mathrm{Si}, 17-21 \mathrm{~nm}$. Итерационная процедура напыления трех-стэкового $\mathrm{Mo} / \mathrm{Si}$ широкополосного зеркала для диапазона 17-21 nm проиллюстрирована на рис. 3. При первом напылении кривая отражения далека от „полочки“, дифрактометрия в жестком диапазоне выявила заметное отклонение толщин зеркала от расчетных (табл. 1), однако последовательно проведенная процедура коррекции позволила за три итерации добиться „плато“ в области 17-21 nm. Плотность Мо составила 0.98 от табличного значения, плотность $\mathrm{Si}$ табличная. Значения шероховатостей для разных ПМЗ в составе стековой структуры составили $\sigma=0.5-0.9 \mathrm{~nm}$ (Mo-на-Si) и $\sigma=0.2-0.5 \mathrm{~nm}$ (Si-на-Мо). Обращает на себя внимание чувствительность кривой отражения к изменениям толщин пленок порядка $1 \AA$. В то же время незначительные отличия плотностей и значений шероховатости не приводят к заметному ухудшению вида кривой отражения, что важно с точки зрения решения прямой задачи, т. е. поиска оптимальных параметров СМЗ.

$\mathrm{Mo} / \mathrm{Si}, \quad 28-33 \mathrm{~nm}$. Для диапазона 28-33 nm было рассчитано теоретически и изготовлено одно $\mathrm{Mo} / \mathrm{Si}$ СМ3, образованное тремя ПМЗ с прослойками Мо и $\mathrm{Si}$ оптимальной толщины (табл. 2). Для удобства напыления эта структура представлена более мелким разбиением на 5 ПМЗ (столбец 2 табл. 2). Спектральная характеристика экспериментального образца приведена на рис. 4, а на рис. 5 - подгонка кривых отражения в жестком и мягком рентгеновских диапазонах в программе Multifitting [17]. Можно видеть, что ошибки в толщинах слоев составили $0.5-1 \mathrm{~nm}$, что будет учтено на следующей стадии работы со структурами данного типа. Плотности $\mathrm{Si}, \mathrm{Mo}$ аналогично СМ3 для 17-21 nm близки к табличным (толщины Мо находятся в диапазоне 5-10 nm, что существенно превышает характерную толщину кристаллизации для Мо), шероховатость также находится в рамках стандартных значений, наблюдается характерная для $\mathrm{Mo} / \mathrm{Si}$ асимметрия границ.

$\mathrm{Mo} / \mathrm{Be}, 11.1-13.8 \mathrm{~nm}$. Серия измерений коэффициента отражения $R(\lambda)$ 6-стекового Мо/Ве зеркала в диапазоне длин волн 10-15 nm приведена на рис. 6. Несмотря на изрезанный характер как спектра источника ЭУФ-излучения (рис. 2), так и самой кривой отражения, продемонстрирована хорошая воспроизводимость результатов измерений; погрешность составила $0.5 \%$.

Для корректного описания вида кривых отражения (рис. 7), в том числе в области критического угла при $\lambda=0.154 \mathrm{~nm}$, необходимо моделировать образование на поверхности зеркала окисла $\mathrm{BeO}$ толщиной $1.4 \mathrm{~nm}$ и „разбухание“ приповерхностного слоя приблизительно на $1 \mathrm{~nm}$ : вместо толщины $h(\mathrm{Be})=2.72 \mathrm{~nm}$ имеем $h(\mathrm{Be}+\mathrm{BeO})=3.82 \mathrm{~nm}$. Толщины остальных слоев, как показывает анализ, соответствуют расчетным с точностью до $0.15 \mathrm{~nm}$ (табл. 3), плотности материалов табличные, шероховатости составили $\sigma=0.65 \mathrm{~nm}$ (Мо-на-Ве) и $\sigma=0.36 \mathrm{~nm}$ (Ве-на-Мо). Кроме того, для улучшения отражательных характеристик Мо/Ве стековых зеркал в диапазоне $11.1-13.8 \mathrm{~nm}$ в дальнейшем необходимо использовать защитное покрытие.

\section{Выводы и заключение}

Таким образом, в ИФМ РАН разработана методика расчета, изготовления и анализа многослойных структур стекового типа. Созданы и изучены экспериментальные образцы $\mathrm{Mo} / \mathrm{Si}$ и $\mathrm{Mo} / \mathrm{Be}$, представляющие интерес для диагностики плазмы.

Для случая, когда была произведена последовательная корректировка технологических параметров, продемонстрировано хорошее совпадение теории и эксперимента. В течение трех коррекций задача была решена, что позволяет изготавливать широкополосные зеркала стекового типа для различных приложений рентгеновской оптики.

\section{Финансирование работы}

Работа выполнена в рамках Программы Президиума РАН I.1 „Экстремальные световые поля и их взаимодействие с веществом“. Часть работ поддержана Российским фондом фундаментальных исследований: разработка Multifitting поддержана грантом № 18-32-00173, напыление экспериментальных образцов грантом 17-52-150006, измерение коэффициентов отражения на длине волны $0.154 \mathrm{~nm}$ - грантом № 18-32-00671.

\section{Конфликт интересов}

Авторы заявляют, что у них нет конфликта интересов. 


\section{Список литературы}

[1] Апериодические элементы в оптике мягкого рентгеновского диапазона / Под ред. Е.Н. Рагозина. М.: Физматлит, 2018. $131 \mathrm{c}$.

[2] Shestov V., Ulyanov S., Vishnyakov E. // SPIE. 2002. Vol. 9144. P. 91443G1.

[3] Yulin S. // SPIE. 2002. Vol. 4782. P. 196-203.

[4] Вишняков Е.А., Каменеи Ф.Ф., Кондратенко В.В. // Квант. электрон. 2012. Т. 42. № 2. С. 143-152

[5] Joensen K.D., Gorenstein P., Wood J.L., Christensen F.E. // Proc. SPIE. 1994. Vol. 2279. P. 180-189.

[6] Kozhevnikov I.V., Bukreeva I.N., Ziegler E. // Nucl. Instrum. Meth. Phys. Res. A. 2001. Vol. 460. N 2-3. P. 424-443.

[7] Ziegler E., Bukreeva I.N., Kozhevnikov I.V., Pirozhkov A.S., Ragozin E.N. // Proc. SPIE. 1999. Vol. 3737. P. 386-395.

[8] Бейгман И.Л., Пирожсков А.С., Рагозин Е.Н. // Письма в ЖЭТФ. 2001. Т. 74. № 3. С.167-171.

[9] Гайкович П.К., Полковников В.Н., Салащенко Н.Н., Чхало Н.И., Шеферс Ф., Соколов А. // Квант. электрон. 2016. T. 46. № 5. C. 406-413.

[10] Kuhlmann T., Yulin S., Feigl T., Kaiser N., Bernitzki H., Lauth H. // Proc. SPIE. 2002. Vol. 4688. P. 509-515.

[11] Ахсахалян А.Д., Клюенков Е.Б., Лопатин А.Я. и др. // Поверхность. Рент., синхр. и нейтр. исслед. 2017. № 1. C. 5-24.

[12] Banine V., Benschop J.P., Leenders M., Moors R. // Proc. SPIE. The International Society for Optical Engineering. 2002. Vol. 3997. P. 126-135.

[13] Braun S., Mai H., Moss M., Scholz R., Leson A. // Jpn. J. Appl. Phys. 2002. Vol. 41. P. 4074-4081.

[14] Svechnikov M.V., Chkhalo N.I., Gusev S.A., Nechay A.N., Pariev D.E., Pestov A.E., Polkovnikov V.N., Tatarskiy D.A., Salashchenko N.N., Schäfers F., Sertsu M.G., Sokolov A., Vainer Y.A., Zorina M.V. // Opt. Express. 2018. Vol. 26. N 26. P. 33718-33731.

[15] Chkhalo N.I., Gusev S.A., Nechay A.N., Pariev D.E., Polkovnikov V.N., Salashchenko F., Schäfers N.N., Sertsu M.G., Sokolov A., Svechnikov M.V., Tatarsky D.A. // Opt. Lett. 2017. Vol. 42. N 24. P. 5070-5073.

[16] Svechnikov M., Pariev D., Nechay A., Salashchenko N., Chkhalo N., Vainer Y., Gaman D. // J. Appl. Cryst. 2017. Vol. 50. P. $1428-1440$.

[17] Свечников М.В. // Нанофизика и наноэлектроника. Матер. XXIII Междунар. симпозиума. 2019. Т. 1. С. 519-520.

[18] Windt D. // Computers in Physics. 1998. Vol. 12. N 4. P. $360-370$.

[19] Kuang S., Li S., Yang H., Huo T., Zhou H. // Opt. Precision Engineer. 2018. Vol. 26. N 10. P. 2395-2406.

[20] Yao Y., Kunieda H., Wang Z. // Opt. Express. 2013. Vol. 21. N 7. P. $8638-8651$.

[21] Барышева М.М., Вайнер Ю.А., Грибков Б.А., Зорина М.В., Пестов А.Е., Рогачев Д.Н., Салащенко Н.Н., Струля И.Л., Чхало Н.И. // Изв. РАН. Сер. Физ. 2011. Т. 75. № 1. C. $71-76$.

[22] Andreev S.S., Akhsakhalyan A.D., Bibishkin M.A., Chkhalo N.I., Gaponov S.V., Gusev S.A., Kluenkov E.B., Prokhorov K.A., Salashchenko N.N., Schäfers F., Zuev S.Yu. // Centr. Europ. J. Phys. 2003. Vol. 1. P. 191-209.

[23] Гарахин С.А., Нечай А.Н., Чхало Н.И., Салащенко Н.Н., Зуев С.Ю., Забродин И.Г., Каськов И.А., Пестов А.Е., Полковников В.Н. // Нанофизика и наноэлектроника. Матер. XXIII Междунар. симпозиума. 2019. Т. 1. С. 447-448. 